Article

\title{
Postprandial Effect of Yogurt Enriched with Anthocyanins from Riceberry Rice on Glycemic Response and Antioxidant Capacity in Healthy Adults
}

\author{
Tanisa Anuyahong ${ }^{1}$, Charoonsri Chusak ${ }^{1}$, Thavaree Thilavech ${ }^{2}$ and Sirichai Adisakwattana ${ }^{1, *(C)}$ \\ 1 Phytochemical and Functional Food Research Unit for Clinical Nutrition, Department of Nutrition and \\ Dietetics, Faculty of Allied Health Science, Chulalongkorn University, Bangkok 10330, Thailand; \\ Tanisa.anuyahong@gmail.com (T.A.); charoonsri.c@gmail.com (C.C.) \\ 2 Department of Food Chemistry, Faculty of Pharmacy, Mahidol University, Bangkok 10400, Thailand; \\ Thavaree.thi@mahidol.ac.th \\ * Correspondence: Sirichai.a@chula.ac.th; Tel.: +66-2-218-1099 (ext. 111)
}

Received: 18 July 2020; Accepted: 23 September 2020; Published: 24 September 2020

\begin{abstract}
The pigment of riceberry rice has been reported to contain anthocyanins which act as a free radical scavenger and inhibitor of carbohydrate digestive enzymes. Since the probiotic yogurt incorporated with the pigment of riceberry rice extract was previously developed, the present study was aimed to investigate the acute effect of riceberry rice yogurt consumption on postprandial glycemic response, antioxidant capacity, and subjective ratings in healthy adults. In a cross-over design, 19 healthy participants were randomized to consume $350 \mathrm{~g}$ of yogurt supplemented with $0.25 \%(w / w)$ riceberry rice extract or the control yogurt. Postprandial plasma glucose, antioxidant status, and subjective ratings were measured at fasting and intervals $(0-3 \mathrm{~h})$ after ingestion of yogurt. The primary outcome was glycemic response; the secondary outcomes were plasma antioxidant capacity. In comparison to the yogurt control, riceberry rice yogurt reduced plasma glucose concentration after $30 \mathrm{~min}$ of consumption. The incremental area under the curve (iAUC) was significantly lower after riceberry rice yogurt load than after the control yogurt load. The consumption of riceberry yogurt caused an acute increase in plasma ferric reducing ability of plasma (FRAP), Trolox equivalent antioxidant capacity (TEAC), and oxygen radical absorbance capacity (ORAC) from the baseline values after $60 \mathrm{~min}$ of $0.25 \pm 0.06 \mathrm{mM} \mathrm{FeSO}_{4}, 253.7 \pm 35.5 \mathrm{mM}$ Trolox equivalents, and $166.8 \pm 28.9 \mathrm{mM}$ Trolox equivalents, respectively. Furthermore, the iAUCs for FRAP, TEAC, ORAC, and protein thiol were higher in riceberry yogurt consumption compared with the control yogurt (1.6-, 1.6-, 2.9-, and 1.9-fold, respectively). A decrease in iAUC for plasma malondialdehyde (MDA) concentration was also observed in the riceberry yogurt group. However, consumption of riceberry rice yogurt and control yogurt showed similar subjective rating scores of hunger, desire to eat, fullness, and satiety. In conclusion, acute consumption of riceberry rice yogurt suppressed postprandial glucose level and improved plasma antioxidant capacity in healthy volunteers.
\end{abstract}

Keywords: yogurt; riceberry rice; postprandial; glycemia; antioxidant; anthocyanin

\section{Introduction}

Yogurt, a semisolid fermented milk produced by lactic acid bacteria, is considered as an important functional food because of high nutritional content such as protein, calcium, vitamin B, phosphorus, magnesium, and potassium [1]. Recent studies reveal that consumption of yogurt fermented by lactic acid bacteria improves gastrointestinal health mediated through gut microflora, bowel transit, and immune response [2]. Especially, probiotic bacteria in yogurt have beneficial effects on host's health by conferring protection against pathogenic bacteria and the prevention of gastrointestinal disorder such 
as irritable bowel syndrome (IBS), acute infectious diarrhea, or food intolerance [3,4]. In long-term intervention studies, the consumption of yogurt has been demonstrated to reduce the risk of developing metabolic diseases such as type 2 diabetes and cardiovascular diseases $[5,6]$. Interestingly, an intake of probiotic yogurt markedly improved glycemic control and increased antioxidant status in patient with type 2 diabetes [7].

In recent years, the role of certain plant-based foods on health benefit has drawn attention to consumers. Following this trend, several attempts have been made to the manufacture of yogurt containing the natural extract from fruits and vegetables in order to improve its nutritional, biological, and sensory properties [8]. For example, addition of cherries, berries, and grapes to yogurts increased the content of bioactive compounds such as phenolic acids and polyphenols [8,9]. Moreover, yogurt fortified with anthocyanins from chokeberries increased antioxidant activity and improved acceptable sensory properties [10]. Interestingly, combination of yogurt and plant-based foods also enhanced proliferation and survival of probiotics and modulated human microbiome [11,12].

Riceberry rice (Oryza sativa L.), a grain covered with deep-purple pigment, contains many nutrient components and bioactive compounds including anthocyanins, mainly cyanidin-3-glucoside (C3G) and peonidin-3-glucoside (P3G) [13]. It has been shown that the extract from riceberry rice possesses various biological activities such as antioxidant, anti-hyperglycemic, anti-hyperlipidemic, anti-glycation, and anti-inflammatory activities $[13,14]$. Consumption of riceberry rice bread had lower glycemic response with increased antioxidant capacity in healthy subjects when compared to Hom mali rice bread [15]. The probiotic yogurt enriched with anthocyanin-rich extract from riceberry rice was successfully developed and improved its functionality by increasing the amount of total phenolic compounds and antioxidant activity [16]. However, there is a substantial lack of evidence on postprandial glycemic response and antioxidant capacity of riceberry rice yogurt consumption in humans. Therefore, this study was aimed to investigated whether the consumption of riceberry rice yogurt affect the level of postprandial plasma glucose in healthy volunteers. The study was also investigated the effect of riceberry rice yogurt on plasma antioxidant capacity, the marker of lipid peroxidation and subjective appetite sensations.

\section{Materials and Methods}

\subsection{Ethical Approval}

The study was approved by the office of Ethics Review Committee for Research Involving Human Research Subjects, Human Science Group, Chulalongkorn University (COA No. 241/2018). The study began in January 2019 and completed in March 2019. The trial was registered at the Thai Clinical Trials Registry (study ID: TCTR20190118006). All subjects gave their written informed consent to participate. All information of participants was kept confidential. There were no major changes in the study protocol after initiation of the study.

\subsection{Riceberry Rice Yogurt}

The set-type yogurt was obtained from the report of Anuyahong et al. [16]. The ingredients consisted of whole milk, skimmed milk powder $(3 \% w / w)$, and sucrose $(5 \% w / w)$, with or without the anthocyanin-rich extract of riceberry rice $(0.25 \% w / w)$. The yogurt contained Streptococcus thermophilus, Lactobacillus delbrueckii subsp. bulgaricus, Lactobacillus acidophilus LA-5 and Bifidobacterium animalis subsp. lactis BB-12. The proximate analysis of yogurt was performed by The Food Research and Testing Laboratory, Faculty of Science, Chulalongkorn University. The nutritional and phytochemical composition are reported in Table 1. 
Table 1. The nutritional composition and phytochemical compounds of yogurt for one serving size (350 g).

\begin{tabular}{ccc}
\hline Composition & Control Yogurt & Riceberry Rice Yogurt \\
\hline Energy (kcal) & 349.1 & 349.6 \\
Carbohydrate (g) & 43.5 & 43.9 \\
Total dietary fiber & n.d & n.d \\
Protein (g) & 13.8 & 13.8 \\
Fat (g) & 13.3 & 13.2 \\
Moisture (g) & 275.9 & 275.5 \\
Ash (g) & 3.5 & 3.6 \\
Total polyphenol content & 16.1 & 28.1 \\
Anthocyanins & n.d & 17.4 \\
Cyanidin-3-glucoside & n.d & 7.9 \\
Peonidin-3-glucoside &
\end{tabular}

n.d = not detected

\subsection{Participants}

Twenty-three subjects were recruited to participate in this study. The inclusion questionnaire was used to evaluate the eligibility criteria of participants. The inclusion criteria were as follow: (1) age of 18-40 years; (2) body mass index (BMI) of $18.5-22.9 \mathrm{~kg} / \mathrm{m}^{2}$; (3) fasting blood glucose level < $100 \mathrm{mg} / \mathrm{dL}$; (4) total cholesterol level < $200 \mathrm{mg} / \mathrm{dL}$; (5) triglyceride level < $150 \mathrm{mg} / \mathrm{dL}$; and (6) physically active. They were excluded if they met any of the following criteria: (1) pregnant or lactating; (2) presence of diabetes mellitus or insulin resistance; (3) use of dietary supplement or medication know to interfere with glucose homeostasis; (4) self-report of alcohol or tobacco products; and (5) allergy or intolerance to yogurt or dairy products. From this group, 23 subjects met the inclusion criteria.

\subsection{Study Design}

The study was a randomized-crossover trial with a one-week washout period. The subjects were randomly assigned to the ingestion of yogurt supplemented with or without $0.25 \%$ riceberry rice extract according to a sequence of random numbers, obtained from the online random number generator (www.randomizer.org). The process of randomization was performed by a principle researcher with a concealed allocation design. The participants were instructed not to take foods high in phytochemicals at least three days before each study period. Additionally, they were also asked to maintain stable habitual dietary intake and activity throughout their participation in the study period and to refrain from alcohol intake and heavy exercise for $24 \mathrm{~h}$ before each test. The primary outcome was glycemic response (postprandial glucose). The secondary outcomes were plasma antioxidant capacity (the ferric reducing ability of plasma, the Trolox equivalent antioxidant capacity, the oxygen radical absorbance capacity, the thiol group, and plasma malondialdehyde).

On the day of testing, the participants arrived at the Department of Nutrition and Dietetics, Faculty of Allied Health Sciences, Chulalongkorn University after overnight fast. Anthropometrics (body weight and height) were measured upon arrival at each session. Following baseline measurements, the fasting blood sample was collected from forearm vein with intravenous catheter by a registered nurse. Thereafter, the subjects were asked to the rate of hunger, fullness, desire to eat, and satiety with a visual analog scale (VAS) rating using a 10-cm scale from 0 ("not at all") to 10 ("extremely") at 0 min (before). They completely consumed $350 \mathrm{~g}$ of the study yogurt within a 10-min period. Blood samples were drawn at 15,30,60,90,120,150, and $180 \mathrm{~min}$, while subjective ratings were taken at 30, 60, 90, 120,150 , and 180 min postprandially. The subjects were restricted to drink water $(<500 \mathrm{~mL})$ during the study. 


\subsection{Biochemical Analysis}

The blood samples were collected into blood colleting tube containing sodium fluoride and EDTA as anticoagulants for the measurement of glucose and antioxidant capacity, respectively. The plasma samples were separated by centrifuged at $3000 \mathrm{rpm}$ for $15 \mathrm{~min}$ at $4{ }^{\circ} \mathrm{C}$ and kept at $-80^{\circ} \mathrm{C}$ for further analysis. The level of plasma glucose concentration was measured by glucose oxidase assay according to the manufacturer's protocol (Glucose Liquicolor, HUMAN GmbH, Wiesbaden, Germany).

The ferric reducing ability of plasma (FRAP) assay in a redox-linked colorimetric reaction was performed according to a previous published report [17]. In brief, the plasma sample (10 $\mu \mathrm{L})$ was mixed with $90 \mu \mathrm{L}$ of freshly prepared FRAP reagent ( $0.3 \mathrm{M}$ sodium acetate buffer (pH 3.6), $10 \mathrm{mM}$ 2,4,6-Tri(2-pyridyl)-s-triazine (TPTZ) in $40 \mathrm{mM} \mathrm{HCl}$, and $20 \mathrm{mM} \mathrm{FeCl}_{3}$ ). After incubation for $30 \mathrm{~min}$ at room temperature, the absorbance was read at $595 \mathrm{~nm}$. The results were expressed as the EC (Equivalence concentration) value obtained from a standard curve of $\mathrm{FeSO}_{4}$.

The Trolox equivalent antioxidant capacity (TEAC) assay was measured using 2,2'-azinobis (3-ethyl benzothiazoline-6-sulfonic acid) (ABTS) radical $\left(\mathrm{ABTS}^{\bullet+}\right.$ ) [15], which was prepared by mixing $7 \mathrm{mM}$ ABTS in $0.1 \mathrm{M}$ PBS ( $\mathrm{pH} 7.4$ ) together with $2.45 \mathrm{mM} \mathrm{K}_{2} \mathrm{~S}_{2} \mathrm{O}_{8}$ in distilled water (1:1, v/v). After incubation for $16 \mathrm{~h}$ at room temperature, the $\mathrm{ABTS}^{\bullet+}$ solution was diluted with $0.1 \mathrm{M} \mathrm{PBS}$ (pH 7.4) to adjust the absorbance between 0.9 and 1.0 at $734 \mathrm{~nm}$. The plasma $(10 \mu \mathrm{L})$ was incubated with the adjusted $\mathrm{ABTS}^{\bullet+}$ solution $(90 \mu \mathrm{L})$ for $6 \mathrm{~min}$ at the room temperature. The absorbance was recorded at $734 \mathrm{~nm}$. The result of plasma TEAC was expressed as mM Trolox equivalents.

The oxygen radical absorbance capacity (ORAC) assay was performed according to a previous report [18]. Briefly, the $10 \times$ dilution of plasma in $0.1 \mathrm{M}$ phosphate buffer saline (PBS), pH 7.4 $(25 \mu \mathrm{L})$ was incubated with $4.8 \mathrm{nM}$ sodium fluorescein in $75 \mathrm{mM}$ PBS $(150 \mu \mathrm{L})$ at $37^{\circ} \mathrm{C}$. After $10 \mathrm{~min}$ of incubation, $64 \mathrm{mM}$ AAPH $(25 \mu \mathrm{L})$ was added to the mixture. The fluorescence intensity was measured for $1 \mathrm{~h}$ with 2-min interval at excitation $485 \mathrm{~nm}$ and emission $535 \mathrm{~nm}$. The ORAC value was calculated from the area under the curve (AUC) and expressed as $\mu$ mol Trolox equivalents.

The thiol group level in plasma was measured using an Ellman's assay [19] with slight modification. In brief, plasma sample $(90 \mu \mathrm{L})$ was mixed with $130 \mu \mathrm{L}$ of $2.5 \mathrm{mM}$ of 5,5'-dithiobis-(2-nitrobenzoic acid) (DTNB) in 0.1 M PBS, pH 7.4 and incubated for $15 \mathrm{~min}$ at the room temperature. Then, the absorbance was measured at $410 \mathrm{~nm}$. The plasma thiol level was calculated and expressed as $\mu \mathrm{M}$ L-cysteine equivalent.

Plasma malondialdehyde (MDA), a lipid peroxidation product, was quantified using thiobarbituric acid reactive substances (TBARS) assay. The plasma sample $(200 \mu \mathrm{L})$ was mixed with trichloroacetic acid $(10 \% w / v)$ and $50 \mathrm{mM}$ 2,6-Di-tert-bytyl-4-methylphenol (BHT) and centrifuged at 12,000 rpm for $10 \mathrm{~min}$ [15]. The supernatant $(200 \mu \mathrm{L})$ was separated and further mixed with $0.67 \%$ TBA before boiling at $100{ }^{\circ} \mathrm{C}$ for $10 \mathrm{~min}$. After cooling down to room temperature, the absorbance of pink-colored of reaction was measured at $532 \mathrm{~nm}$. Plasma MDA concentration was calculated from the calibration curve of MDA and expressed as $\mu \mathrm{mol} / \mathrm{L}$ MDA.

\subsection{Sample Size}

The sample size was calculated following a previous study of postprandial response that reported a significant change in AUC for plasma glucose with a study power of $80 \%$ and alpha of 0.05 [20]. The sample size of 17 subjects were calculated with a confidence level of $95 \%(\alpha=5 \%)$ and power of $80 \%$. Considering the $30 \%$ dropout, the final sample size was increased to 23 subjects.

\subsection{Statistical Analysis}

The results are expressed as mean \pm SEM. The normality and homogeneity of the data was tested by Shapiro-Wilk test. Repeated-measures ANOVA was performed to determine the effect of treatment, time, and interaction of treatment and time followed by a paired $t$ test to find the significance for each time point at $p<0.05$. The incremental area under the curve (iAUC) for postprandial glucose, 
antioxidant status and lipid peroxidation (MDA) was calculated by using the trapezoidal rule integrated count areas above and below the fasting baseline concentration. Paired samples t-tests confirmed significant differences in the results from iAUC $(p<0.05)$.

\section{Results}

\subsection{Participants}

Twenty-three participants were recruited at the beginning and only nineteen participants (8 male and 11 female) completed the study. Four participants who did not receive intervention were excluded from analysis. The recruitment and enrollment data are presented in Figure 1. The characteristics of participants are reported in Table 2 .

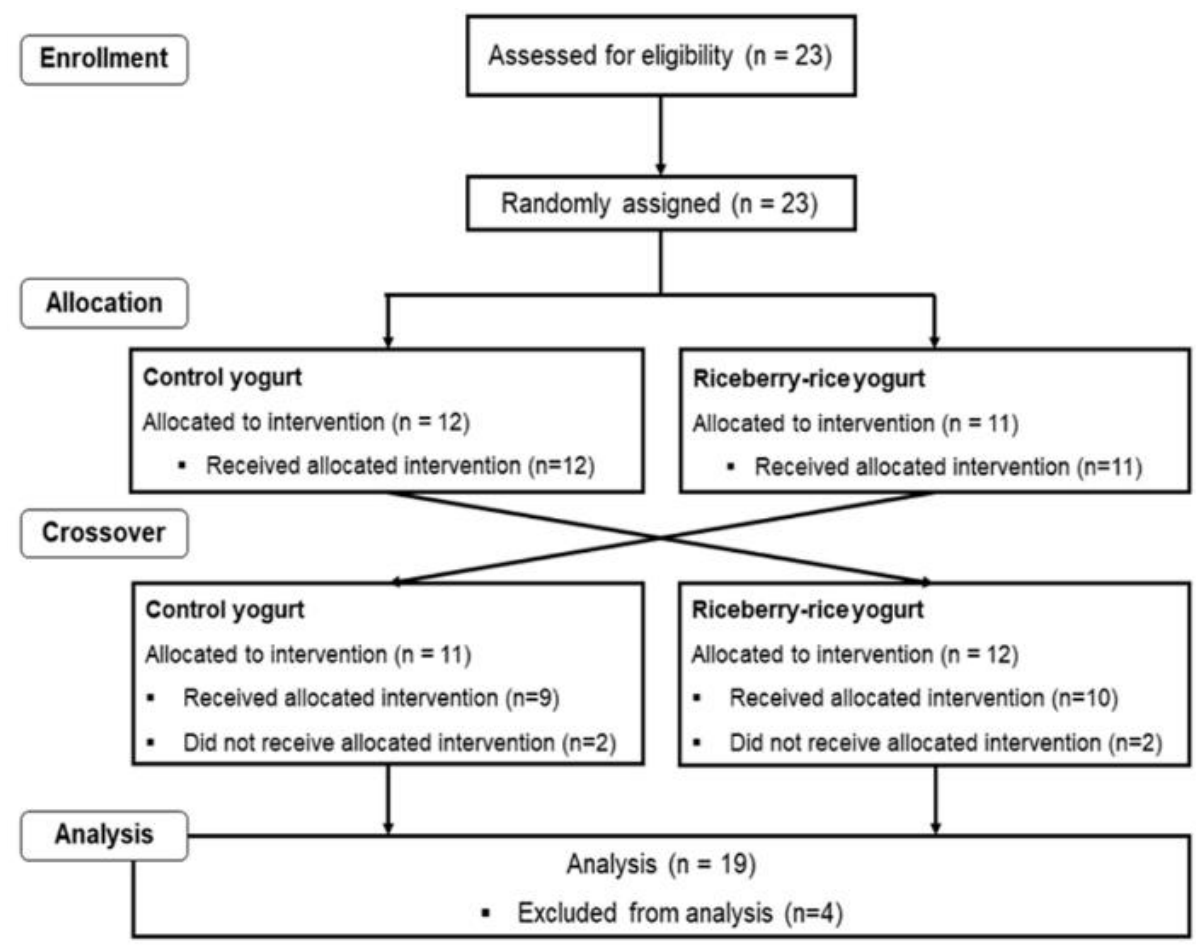

Figure 1. Consolidating Standards of Reporting (CONSORT) flow diagram of selection of study participants.

Table 2. Baseline characteristics of participants.

\begin{tabular}{lc}
\hline \multicolumn{1}{c}{ Parameters } & Mean \pm SEM \\
\hline Age (years) & $28.1 \pm 3.0$ \\
Height $(\mathrm{m})$ & $1.65 \pm 0.1$ \\
Weight $(\mathrm{kg}$ & $58.3 \pm 2.2$ \\
BMI $\left(\mathrm{kg} / \mathrm{m}^{2}\right)$ & $21.2 \pm 0.4$ \\
Fasting plasma glucose $(\mathrm{mg} / \mathrm{dL})$ & $83 \pm 1.0$ \\
Total cholesterol $(\mathrm{mg} / \mathrm{dL})$ & $167.3 \pm 8.1$ \\
Serum triglyceride $(\mathrm{mg} / \mathrm{dL})$ & $53.0 \pm 3.8$ \\
LDL-C (mg/dL) & $113.5 \pm 6.6$ \\
HDL-C (mg/dL) & $43.2 \pm 3.5$ \\
Creatinine $(\mathrm{mg} / \mathrm{dL})$ & $0.7 \pm 0.1$ \\
Blood urea nitrogen $(\mathrm{BUN})(\mathrm{mg} / \mathrm{dL})$ & $11.6 \pm 0.5$ \\
\hline
\end{tabular}

All values are mean $\pm \operatorname{SEM}, n=19$ 


\subsection{Postprandial Plasma Glucose}

Incremental changes in postprandial plasma glucose concentration after consumption of yogurts are demonstrated in Figure 2A. The peak of plasma glucose level was at 15 and $30 \mathrm{~min}$ after consumption of all yogurts. Postprandial plasma glucose concentration following riceberry yogurt was significantly lower than following the control at 30 and $120 \mathrm{~min}$. As shown in Figure 2B, a reduction in iAUC of glucose for riceberry rice yogurt relative to the control was observed.
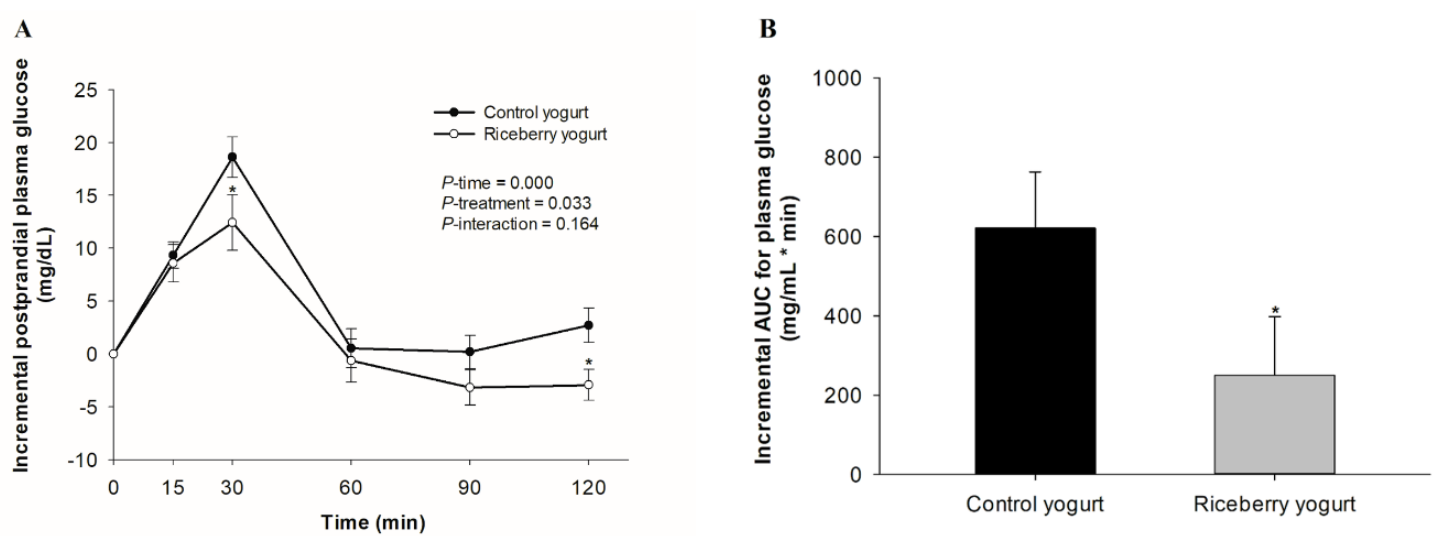

Figure 2. Incremental changes in postprandial plasma glucose concentration (A) and the incremental area under the curve (iAUC) for postprandial plasma glucose (B) in healthy participants after consuming either the control yogurt $(\bullet)$ or riceberry rice yogurt $(\bigcirc)$. Data are presented as mean \pm SEM, $n=19$. * $p<0.05$ compared to the control yogurt.

\subsection{Postprandial Plasma Antioxidant Status}

Compared with the control, the incremental changes in postprandial FRAP was significantly increased at 60, 90, and 120 min after consumption of riceberry rice yogurt (Figure 3A). The results show that consumption of riceberry rice yogurt resulted 1.6-fold greater in the iAUC of plasma FRAP level when compared with the control (Figure 3B).

Consumption of all yogurts caused a significant increase in TEAC above baseline for all time points. Plasma TEAC level at 30, 90, 120, and 150 min was significantly higher in participants who received riceberry rice yogurt than in those who consumed the control yogurt (Figure 3C). The iAUC of postprandial plasma TEAC was 1.6-fold greater in riceberry rice yogurt than in the control (Figure 3D).

Incremental changes in postprandial plasma ORAC after consumption of yogurts are presented in Figure 3E. The postprandial plasma ORAC level appeared immediately following intake of all yogurts and returned to the baseline level at $180 \mathrm{~min}$. The plasma ORAC level was significantly higher for riceberry rice yogurt than the control at 30,60, 90, and $120 \mathrm{~min}$. In particular, the 2.9-fold increase in iAUC of plasma ORAC was observed for the subjects who consumed riceberry rice yogurt (Figure 3F).

Figure $3 \mathrm{G}$ presents incremental changes in postprandial plasma thiol after ingestion of yogurts. The postprandial plasma thiol level was elevated after consumption of all yogurts when compared to the baseline level. The results show that the postprandial plasma thiol level did not differ between riceberry rice yogurt and the control at each time point. Nevertheless, riceberry rice yogurt caused a 1.8-fold increase iAUC of plasma thiol, in comparison to the control yogurt (Figure 3H).

Incremental changes in postprandial plasma MDA concentration after consumption of yogurts are shown in Figure 3I. Plasma MDA concentration increased significantly from baseline following the control yogurt. Interestingly, consumption of riceberry rice yogurt markedly reduced the rise in plasma MDA concentration at 60, 90, and $180 \mathrm{~min}$. A reduction in iAUC of postprandial plasma MDA (33\%) was perceived following consumption of riceberry rice yogurt (Figure 3J). 

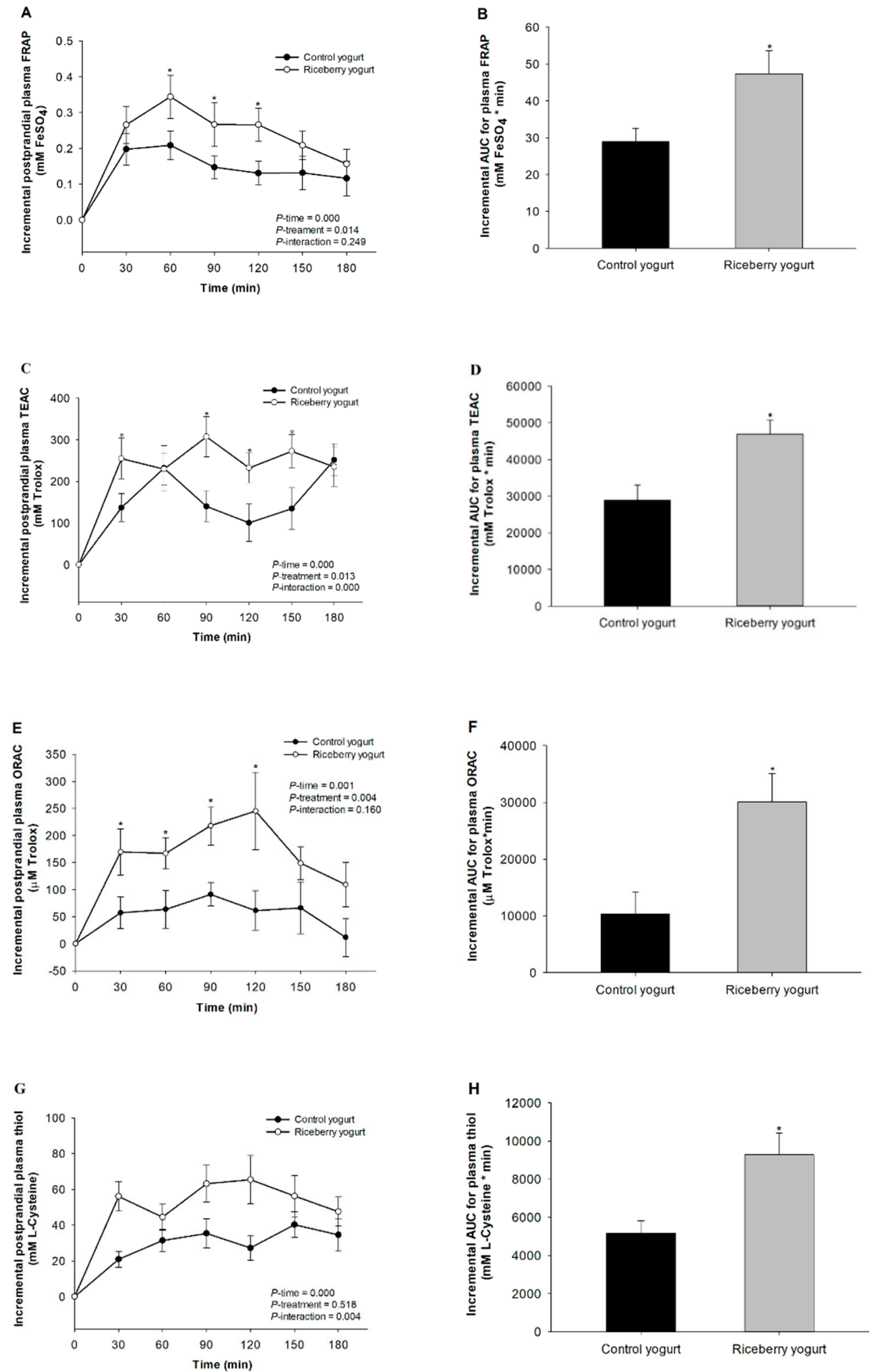

Figure 3. Cont. 

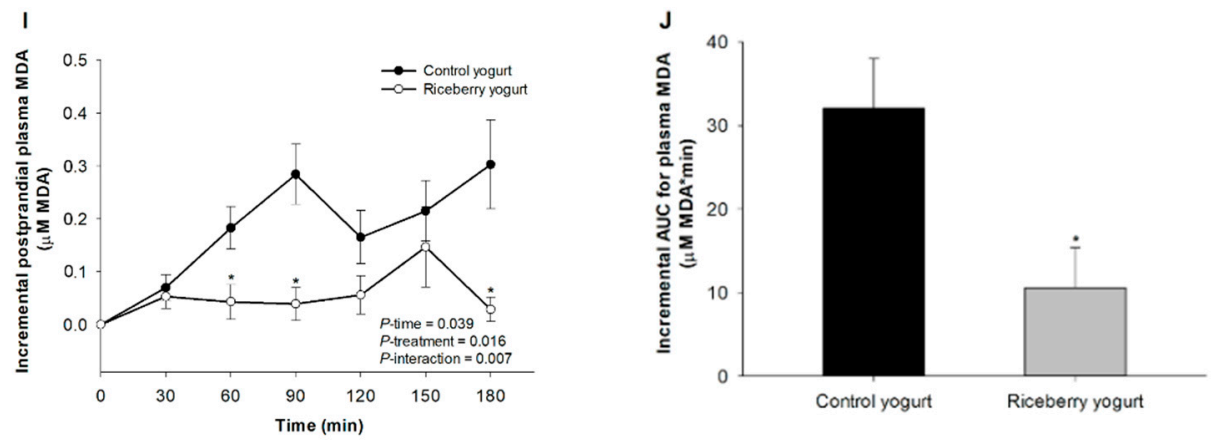

Figure 3. Incremental changes in postprandial plasma: ferric reducing ability of plasma (FRAP) (A); Trolox equivalent antioxidant capacity (TEAC) (C); oxygen radical absorbance capacity (ORAC) (E); thiol (G); and malondialdehyde concentration (MDA) (I). The incremental area under the curve (iAUC) for postprandial plasma: FRAP (B); TEAC (D); ORAC (F); thiol (H); and MDA (J) in healthy participants after consuming either the control yogurt $(\bullet)$ or riceberry rice yogurt $(\bigcirc)$. Data are presented as mean \pm $\mathrm{SEM}, n=19 .{ }^{*} p<0.05$ compared to the control yogurt.

\subsection{Subjective Rating}

The subjective rating scores of hunger, fullness, desire to eat, and satiety after consumption of yogurts are illustrated in Figure 4A-D. All yogurts markedly reduced the score of hunger and desire to eat and increased the score of satiety and fullness after $30 \mathrm{~min}$ of ingestion as compared to baseline. However, there were no statistically significant differences in the rating score of hunger, fullness, desire to eat, and satiety among all yogurts.
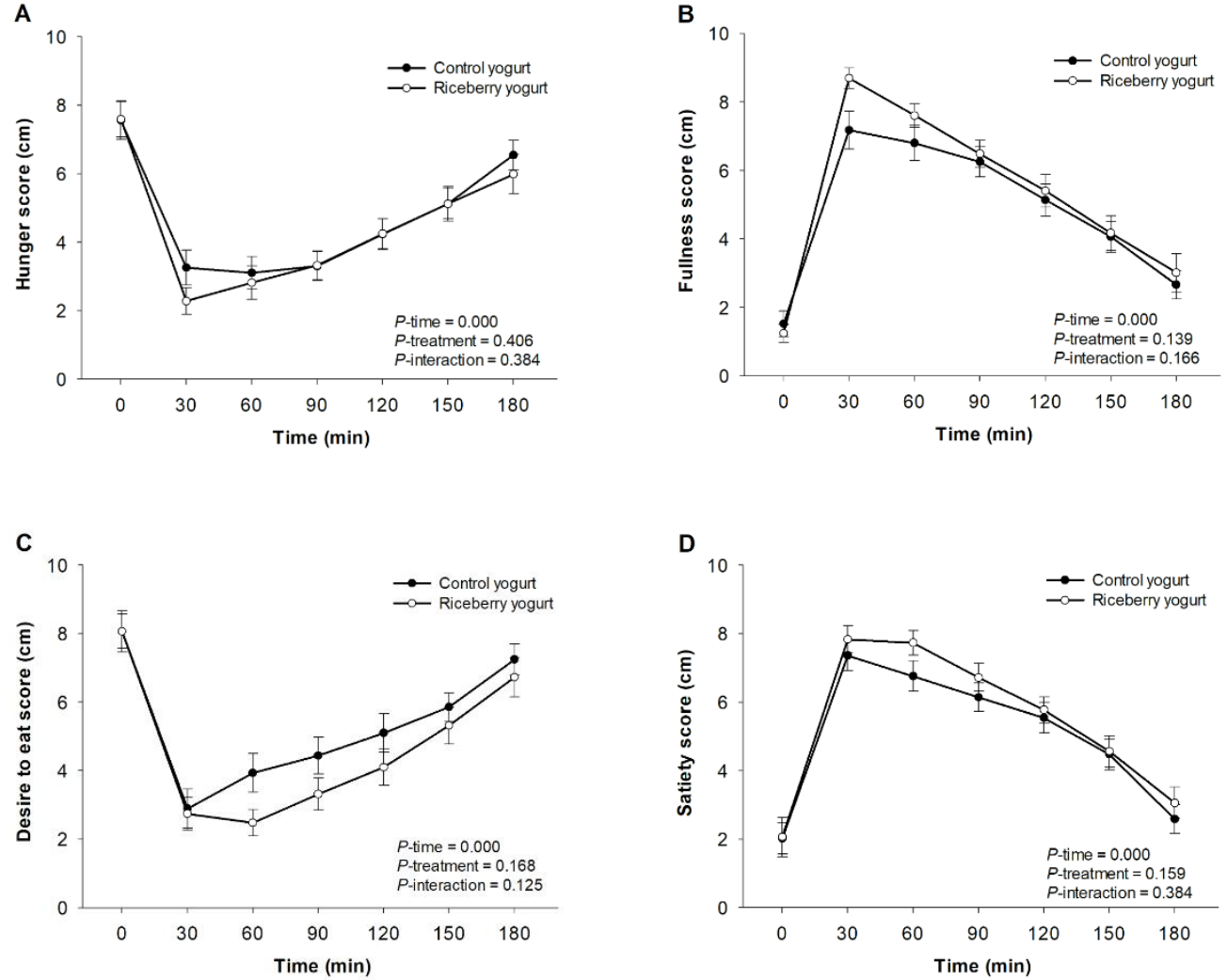

Figure 4. Incremental changes in subjective ratings for: hunger (A); fullness (B); desire to eat (C); and satiety (D) in healthy participants after consuming either the control yogurt $(\bullet)$ or riceberry rice yogurt $(\bigcirc)$. Data are presented as mean \pm SEM, $n=19$. 


\section{Discussion}

Yogurt has received considerable attention as a potential approach to reduce the risks of weight gain, obesity, type 2 diabetes, and cardiovascular diseases [5,6]. Especially, several studies have reported the successful fortification of yogurt with bioactive compounds from edible plants such as green tea, black tea, white tea [21], chamomile [22], strawberry pulp [23], and aronia juice [10]. In our previous study, the supplementation of probiotic yogurt with anthocyanins from riceberry rice provided bioactive compounds and increased its functionality by increasing total phenolic content (TPC), cyanidin-3-glucoside (C3G), and peonidin-3-glucoside (P3G) concomitant with the elevation of DPPH radical scavenging activity and ferric reducing antioxidant power. In gastrointestinal digestion, this yogurt produced higher release of TPC and FRAP than the control [16]. Therefore, this was the first human study to investigate whether riceberry rice yogurt decreases postprandial glycemic response and improves antioxidant capacity in healthy volunteers. A reduction in the postprandial glucose excursion (40.23\%) after consumption of riceberry rice yogurt was observed in healthy subjects. Cross-over studies have explored acute effects riceberry rice bread (50 g) on postprandial glycemic and insulin response in healthy volunteers [15]. After the riceberry rice bread intake, the AUC was $60 \%$ lower in comparison to jasmine rice bread. Furthermore, it was found to produce an attenuated postprandial insulin concentration after $15 \mathrm{~min}$ of consumption. The main reason to explain these effects is the ability of riceberry rice and its phytochemical compounds to inhibit carbohydrate digestive enzymes $[15,24,25]$. A previous study supports this reason that the anthocyanin-rich extract of riceberry rice was capable of inhibiting intestinal $\alpha$-glucosidase such as maltase and sucrase [14]. Especially, C3G and P3G, the major anthocyanins identified in riceberry rice extract, was proved to be effective pancreatic $\alpha$-amylase and $\alpha$-glucosidase inhibitors $[14,26,27]$. Since lactose from milk was the major source of carbohydrate in the yogurt, a further study is needed to investigate the inhibitory effect of anthocyanins on lactase activity.

Postmeal hyperglycemia and glycemic fluctuations induces excessive production of reactive oxygen species (ROS). The excessive formation of ROS may be a contributing factor for induction of pathological changes related to the development of cardiovascular diseases [28]. Interestingly, dietary antioxidants help scavenge and neutralize excessive and inappropriate ROS, sequencing to balance against oxidant condition [29]. Scientific evidence suggests that consumption of phytochemical-rich plants improved plasma antioxidant capacity and reduced lipid peroxidation in humans [30-32]. The different assays have been commonly used to measure plasma antioxidant capacity such as ORAC, TEAC, and FRAP. The ORAC assay refers the ability of antioxidant molecules to inhibit peroxyl radical induced oxidation [33], whereas the TEAC assay indicates the ability of hydrogen-donating antioxidants to neutralize a radical cation in both lipophilic and hydrophilic environments [34]. FRAP assay could reflect the ability of antioxidants to reduce the reaction of $\mathrm{Fe} 3+/ \mathrm{Fe} 2+$ couple. In addition, protein thiols in plasma has multifaceted functions, including a pivotal role in antioxidant defense [35]. Compared to fasting state, consumption of yogurt control slightly increased plasma antioxidant capacity (FRAP, TEAC, and ORAC) and protein thiol level. This result is entirely due to yogurt containing milk proteins and natural substances which have antioxidant activity [36]. Remarkably, plasma FRAP, TEAC, ORAC, and protein thiol levels were high 180 min after riceberry rice yogurt consumption, in comparison with the yogurt control. Increases in postprandial antioxidant capacity are supported by in vitro studies indicating that yogurt supplemented with riceberry rice extract $(0.25 \%)$ had 4.8 -fold higher FRAP than the control yogurt [16]. Similar results were also reported by Chusak et al. who found a greater increase in plasma FRAP following riceberry rice bread consumption [15]. A notable result from our study was a marked increase in plasma MDA above baseline after an intake of yogurt control, indicating that diet could induce postprandial lipid oxidation. This alteration was noticeably attenuated by riceberry rice yogurt consumption. These findings led us to hypothesize that riceberry rice yogurt would decrease postprandial oxidative stress related to their antioxidant activity.

Other clinical studies have also shown improvement in plasma antioxidant capacity together with reduction of lipid peroxidation following consumption of anthocyanin-rich plants such as butterfly 
pea flower [37], Chilean berry [38], and açai berry [31]. It is suggested that the improvement of plasma antioxidant status may be partly attributed to the antioxidant activity of phenolic compounds [37]. In this context, C3G and P3G, the incorporated active ingredients in riceberry rice yogurt, have been recognized as antioxidant agents as represented by FRAP, TEAC, and ORAC assays [39,40]. In addition, C3G and P3G had the ability to reduce the formation of lipid peroxidation in UVB irradiation model and vitamin E-depleted rat [41,42]. Interestingly, after consumption of anthocyanin-rich strawberries and chokeberries, mainly C3G reduced plasma MDA concentration by $31 \%$ and $46 \%$, respectively $[43,44]$. Through these actions, C3G and P3G in riceberry rice yogurt may play a role in an increase in plasma antioxidant capacity, leading to decrease in lipid peroxidation. However, other phytochemical compounds in riceberry rice may influence postprandial antioxidant capacity. Therefore, the quantification of the postprandial concentration of individual polyphenol after riceberry yogurt consumption is needed, which may help explore the role of riceberry rice yogurt in suppressing postprandial oxidative stress.

Visual analog scales (VAS) are relievable tools for the evaluation of subjective appetite sensation about hunger, fullness, desire to eat, and satiety [45]. In our study, the scores of all parameters did not show any significant differences between riceberry rice yogurt and the control yogurt. This finding is in agreement with the earlier study that bread made from riceberry rice did not alter subjective rating scores of hunger, fullness, desire to eat, and satiety in healthy adults. In addition, no change in postprandial level of glucagon-like peptide-1 (GLP-1), an incretin hormone, was detected following consumption of riceberry rice bread [15]. GLP-1 is responsible for the stimulation of insulin secretion, inhibition of glucagon secretion and gastric emptying, and regulation of appetite and satiety [46]. Interestingly, anthocyanins stimulated the secretion of GLP-1 from Murine GLUTag cell line [47]. We hypothesized that consumption of riceberry rice yogurt could not modulate satiety and appetite through the stimulation of GLP-1, possibly as a result from a small amount of anthocyanin in yogurt and its low bioavailability. Furthermore, we acknowledge some potential limitations to the current study. First, we did not introduce a full meal to consume with riceberry yogurt. Other macro- and micronutrients may interfere the postprandial effect of riceberry rice yogurt on plasma glucose and antioxidant capacity. Moreover, this study was only a relatively young and healthy population; older age subjects were not included to increase the homogeneity of postprandial response.

\section{Conclusions}

The present findings indicate that consumption of riceberry rice yogurt had a favorable effect in reducing postprandial plasma glucose and plasma MDA with improvement of plasma antioxidant status. With respect to appetite ratings, no significant change in scores of fullness, desire to eat, and satiety was observed following consumption of riceberry yogurt. The results suggest that riceberry rice yogurt could be a healthy food for improving the postprandial glycemic and antioxidant response in humans. The further study should determine the long-term effect of riceberry rice yogurt consumption in other populations at risk for chronic diseases following meals.

Author Contributions: T.A., Conceptualization, Data curation, Investigation, Methodology, Formal analysis, Validation, and Writing-original draft; C.C., Data curation, Formal analysis, Writing-original draft, and Writing-review and editing; T.T., Writing-review and editing; and S.A., Project administration, Conceptualization, Funding acquisition, Supervision, Writing —original draft, and Writing-review and editing. All authors have read and agreed to the published version of the manuscript.

Funding: This research was supported by Grant for International Research Integration: Chula Research Scholar, Ratchadaphiseksomphot Endowment Fund, Chulalongkorn University (CU-GRS-62-04-37-01).

Acknowledgments: We would like to thank the 90th Anniversary Chulalongkorn University Fund (Ratchadaphiseksomphot Endowment Fund), Graduate school, Chulalongkorn University and Rachadapisek Sompote Fund for Postdoctoral Fellowship, Chulalongkorn University.

Conflicts of Interest: The authors declare no conflict of interest. 


\section{References}

1. Tremblay, A.; Panahi, S. Yogurt consumption as a signature of a healthy diet and lifestyle. J. Nutr. 2017, 147, 1476S-1480S. [CrossRef] [PubMed]

2. Adolfsson, O.; Meydani, S.N.; Russell, R.M. Yogurt and gut function. Am. J. Clin. Nutr. 2004, 80, $245-256$. [CrossRef] [PubMed]

3. Gareau, M.G.; Sherman, P.M.; Walker, W.A. Probiotics and the gut microbiota in intestinal health and disease. Nat. Rev. Gastroenterol. Hepatol. 2010, 7, 503. [CrossRef] [PubMed]

4. Plessas, S.; Bosnea, L.; Alexopoulos, A.; Bezirtzoglou, E. Potential effects of probiotics in cheese and yogurt production: A review. Eng. Life Sci. 2012, 12, 433-440. [CrossRef]

5. Crichton, G.E.; Alkerwi, A. Dairy food intake is positively associated with cardiovascular health: Findings from observation of cardiovascular risk factors in Luxembourg study. Nutr. Res. 2014, 34, 1036-1044. [CrossRef]

6. Possa, G.; Corrente, J.E.; Fisberg, M. Yogurt consumption is associated with a better lifestyle in Brazilian population. BMC Nutr. 2017, 3, 29. [CrossRef] [PubMed]

7. Ejtahed, H.S.; Mohtadi-Nia, J.; Homayouni-Rad, A.; Niafar, M.; Asghari-Jafarabadi, M.; Mofid, V. Probiotic yogurt improves antioxidant status in type 2 diabetic patients. Nutrients 2012, 28, 539-543. [CrossRef]

8. Celik, S.; Bakırcı, I.; Şat, I. Physicochemical and organoleptic properties of yogurt with cornelian cherry paste. Int. J. Food Prop. 2006, 9, 401-408. [CrossRef]

9. Öztürk, B.; Öner, M. Production and evaluation of yogurt with concentrated grape juice. J. Food Sci. 1999, 64, 530-532. [CrossRef]

10. Nguyen, L.; Hwang, E.S. Quality characteristics and antioxidant activity of yogurt supplemented with aronia (Aronia melanocarpa) juice. Prev. Nutr. Food Sci. 2016, 21, 330. [CrossRef]

11. Fernandez, M.A.; Marette, A. Potential health benefits of combining yogurt and fruits based on their probiotic and prebiotic properties. Adv. Nutr. 2017, 8, 155S-164S. [CrossRef] [PubMed]

12. Abdel-Hamid, M.; Romeih, E.; Huang, Z.; Enomoto, T.; Huang, L.; Li, L. Bioactive properties of probiotic set-yogurt supplemented with Siraitia grosvenorii fruit extract. Food Chem. 2020, 303, 125400. [CrossRef] [PubMed]

13. Leardkamolkarn, V.; Thongthep, W.; Suttiarporn, P.; Kongkachuichai, R.; Wongpornchai, S.; Wanavijitr, A. Chemopreventive properties of the bran extracted from a newly-developed Thai rice: The Riceberry. Food Chem. 2011, 25, 978-985. [CrossRef]

14. Poosri, S.; Thilavech, T.; Pasukamonset, P.; Suparpprom, C.; Adisakwattana, S. Studies on Riceberry rice (Oryza sativa L.) extract on the key steps related to carbohydrate and lipid digestion and absorption: A new source of natural bioactive substances. NFS J. 2019, 17, 17-23. [CrossRef]

15. Chusak, C.; Pasukamonset, P.; Chantarasinlapin, P.; Adisakwattana, S. Postprandial glycemia, insulinemia, and antioxidant status in healthy subjects after ingestion of bread made from anthocyanin-rich riceberry rice. Nutrients 2020, 12, 782. [CrossRef]

16. Anuyahong, T.; Chusak, C.; Adisakwattana, S. Incorporation of anthocyanin-rich riceberry rice in yogurts: Effect on physicochemical properties, antioxidant activity and in vitro gastrointestinal digestion. LWT-Food Sci. Technol. 2020, 129, 109571. [CrossRef]

17. Benzie, I.F.; Strain, J.J. The ferric reducing ability of plasma (FRAP) as a measure of "antioxidant power": The FRAP assay. Anal. Chem. 1996, 239, 70-76. [CrossRef]

18. Chusak, C.; Thilavech, T.; Adisakwattana, S. Consumption of Mesona chinensis attenuates postprandial glucose and improves antioxidant status induced by a high carbohydrate meal in overweight subjects. Am. J. Chin. Med. 2014, 42, 315-336. [CrossRef]

19. Ellman, G.L. Tissue sulfhydryl groups. Arch. Biochem. Biophys. 1959, 82, 70-77. [CrossRef]

20. Törrönen, R.; McDougall, G.J.; Dobson, G.; Stewart, D.; Hellström, J.; Mattila, P.; Pihlava, J.M.; Koskela, A.; Karjalainen, R. Fortification of blackcurrant juice with crowberry: Impact on polyphenol composition, urinary phenolic metabolites, and postprandial glycemic response in healthy subjects. J. Funct. Foods 2012, 4, 746-756. [CrossRef]

21. Muniandy, P.; Shori, A.B.; Baba, A.S. Influence of green, white and black tea addition on the antioxidant activity of probiotic yogurt during refrigerated storage. Food Packag. Shelf Life 2016, 8, 1-8. [CrossRef] 
22. Caleja, C.; Barros, L.; Antonio, A.L.; Carocho, M.; Oliveira, M.B.P.; Ferreira, I.C. Fortification of yogurts with different antioxidant preservatives: A comparative study between natural and synthetic additives. Food Chem. 2016, 210, 262-268. [CrossRef] [PubMed]

23. Jaster, H.; Arend, G.D.; Rezzadori, K.; Chaves, V.C.; Reginatto, F.H.; Petrus, J.C.C. Enhancement of antioxidant activity and physicochemical properties of yogurt enriched with concentrated strawberry pulp obtained by block freeze concentration. Food Res. Int. 2018, 104, 119-125. [CrossRef] [PubMed]

24. Adisakwattana, S.; Ruengsamran, T.; Kampa, P.; Sompong, W. In vitro inhibitory effects of plant-based foods and their combinations on intestinal $\alpha$-glucosidase and pancreatic $\alpha$-amylase. BMC Complem. Altern. Med. 2012, 12, 110. [CrossRef] [PubMed]

25. Törrönen, R.; Sarkkinen, E.; Tapola, N.; Hautaniemi, E.; Kilpi, K.; Niskanen, L. Berries modify the postprandial plasma glucose response to sucrose in healthy subjects. Br. J. Nutr. 2010, 103, 1094-1097. [CrossRef] [PubMed]

26. Akkarachiyasit, S.; Charoenlertkul, P.; Yibchok-anun, S.; Adisakwattana, S. Inhibitory activities of cyanidin and its glycosides and synergistic effect with acarbose against intestinal $\alpha$-glucosidase and pancreatic $\alpha$-amylase. Int. J. Mol. Sci. 2010, 11, 3387-3396. [CrossRef]

27. Sui, X.; Zhang, Y.; Zhou, W. In vitro and in silico studies of the inhibition activity of anthocyanins against porcine pancreatic $\alpha$-amylase. J. Funct. Foods 2016, 21, 50-57. [CrossRef]

28. O'Keefe, J.H.; Bell, D.S. Postprandial hyperglycemia/hyperlipidemia (postprandial dysmetabolism) is a cardiovascular risk factor. Am. J. Cardiol. 2007, 100, 899-904. [CrossRef]

29. Stephens, J.W.; Khanolkar, M.P.; Bain, S.C. The biological relevance and measurement of plasma markers of oxidative stress in diabetes and cardiovascular disease. Atherosclerosis 2009, 202, 321-329. [CrossRef]

30. Einbond, L.S.; Reynertson, K.A.; Luo, X.D.; Basile, M.J.; Kennelly, E.J. Anthocyanin antioxidants from edible fruits. Food Chem. 2004, 84, 23-28. [CrossRef]

31. Mertens-Talcott, S.U.; Rios, J.; Jilma-Stohlawetz, P.; Pacheco-Palencia, L.A.; Meibohm, B.; Talcott, S.T.; Derendorf, H. Pharmacokinetics of anthocyanins and antioxidant effects after the consumption of anthocyanin-rich acai juice and pulp (Euterpe oleracea Mart.) in human healthy volunteers. J. Agric. Food Chem. 2008, 56, 7796-7802. [CrossRef] [PubMed]

32. Rizvi, S.I.; Jha, R.; Pandey, K.B. Activation of erythrocyte plasma membrane redox system provides a useful method to evaluate antioxidant potential of plant polyphenols. Methods Mol. Biol. 2010, 594, 341-348. [PubMed]

33. Cao, G.; Alessio, H.M.; Cutler, R.G. Oxygen-radical absorbance capacity assay for antioxidants. Free Radic. Biol. Med. 1993, 14, 303-311. [CrossRef]

34. Mira, L.; Silva, M.; Rocha, R.; Manso, C. Measurement of relative antioxidant activity of compounds: A methodological note. Redox Rep. 1999, 4, 69-74. [CrossRef] [PubMed]

35. Sen, C.K.; Packer, L. Thiol homeostasis and supplements in physical exercise. Am. J. Clin. Nutr. 2000, 72, 653S-669S. [CrossRef] [PubMed]

36. Khan, I.T.; Nadeem, M.; Imran, M.; Ullah, R.; Ajmal, M.; Jaspal, M.H. Antioxidant properties of milk and dairy products: A comprehensive review of the current knowledge. Lipids Health Dis. 2019, 18, 41. [CrossRef]

37. Chusak, C.; Thilavech, T.; Henry, C.J.; Adisakwattana, S. Acute effect of Clitoria ternatea flower beverage on glycemic response and antioxidant capacity in healthy subjects: A randomized crossover trial. BMC Complement. Altern. Med. 2018, 18,1-11. [CrossRef]

38. Urquiaga, I.; Ávila, F.; Echeverria, G.; Perez, D.; Trejo, S.; Leighton, F. A Chilean berry concentrate protects against postprandial oxidative stress and increases plasma antioxidant activity in healthy humans. Oxid. Med. Cell. Longev. 2017, 2017, 8361493. [CrossRef]

39. Stintzing, F.C.; Stintzing, A.S.; Carle, R.; Frei, B.; Wrolstad, R.E. Color and antioxidant properties of cyanidin-based anthocyanin pigments. J. Agric. Food Chem. 2002, 50, 6172-6181. [CrossRef]

40. Wang, Y.; Zhu, J.; Meng, X.; Liu, S.; Mu, J.; Ning, C. Comparison of polyphenol, anthocyanin and antioxidant capacity in four varieties of Lonicera caerulea berry extracts. Food Chem. 2016, 197, 522-529. [CrossRef]

41. Tsuda, T.; Shiga, K.; Ohshima, K.; Kawakishi, S.; Osawa, T. Inhibition of lipid peroxidation and the active oxygen radical scavenging effect of anthocyanin pigments isolated from Phaseolus vulgaris L. Biochem. Pharmacol. 1996, 52, 1033-1039. [CrossRef]

42. Ramirez-Tortosa, C.; Andersen, Ø.M.; Gardner, P.T.; Morrice, P.C.; Wood, S.G.; Duthie, S.J.; Collins, A.R.; Duthie, G.G. Anthocyanin-rich extract decreases indices of lipid peroxidation and DNA damage in vitamin E-depleted rats. Free Radic. Biol. Med. 2001, 31, 1033-1037. [CrossRef] 
43. Alvarez-Suarez, J.M.; Giampieri, F.; Tulipani, S.; Casoli, T.; Di Stefano, G.; González-Paramás, A.M.; Santos-Buelga, C.; Busco, F.; Quiles, J.L.; Cordero, M.D.; et al. One-month strawberry-rich anthocyanin supplementation ameliorates cardiovascular risk, oxidative stress markers and platelet activation in humans. J. Nutr. Biochem. 2014, 25, 289-294. [CrossRef] [PubMed]

44. Kardum, N.; Takić, M.; Šavikin, K.; Zec, M.; Zdunić, G.; Spasić, S.; Konić-Ristić, A. Effects of polyphenol-rich chokeberry juice on cellular antioxidant enzymes and membrane lipid status in healthy women. J. Funct. Foods. 2014, 9, 89-97. [CrossRef]

45. Mortensen, M.W.; Spagner, C.; Cuparencu, C.; Astrup, A.; Raben, A.; Dragsted, L.O. Sea buckthorn decreases and delays insulin response and improves glycaemic profile following a sucrose-containing berry meal: A randomised, controlled, crossover study of Danish sea buckthorn and strawberries in overweight and obese male subjects. Eur. J. Nutr. 2018, 57, 2827-2837. [CrossRef]

46. Tsuda, T. Possible abilities of dietary factors to prevent and treat diabetes via the stimulation of glucagon-like peptide-1 secretion. Mol. Nutr. Food Res. 2015, 59, 1264-1273. [CrossRef]

47. Kato, M.; Tani, T.; Terahara, N.; Tsuda, T. The anthocyanin delphinidin 3-rutinoside stimulates glucagon-like peptide-1 secretion in murine GLUTag cell line via the $\mathrm{Ca}^{2+} /$ calmodulin-dependent kinase II pathway. PLoS ONE 2015, 10, e0126157. [CrossRef]

(C) 2020 by the authors. Licensee MDPI, Basel, Switzerland. This article is an open access article distributed under the terms and conditions of the Creative Commons Attribution (CC BY) license (http://creativecommons.org/licenses/by/4.0/). 